\title{
Estudo bibliometrico sobre ostomia e feridas
}

\author{
Bibliometric study on ostomy and wounds \\ Estudio bibliométrico sobre ostomía y heridas
}

Vitoria Vilas Boas da Silva Bomfim ORCID: https://orcid.org/0000-0003-4897-0279

Centro Universitário Jorge Amado, Brasil E-mail: pesquisaclinica9@gmail.com Andressa Fernandes da Silva ORCID: https://orcid.org/0000-0002-1942-0860 Instituto Nacional de Cardiologia, Brasil E-mail: andressafdurso@gmail.com

Samara Dantas de Medeiros Diniz ORCID: https://orcid.org/0000-0001-9418-0185

Faculdade Estácio do Rio Grande do Norte, Brasil E-mail:samaradantas1998@gmail.com

Renata Porangaba Cavalcante ORCID: https://orcid.org/0000-0002-9408-3251

Centro Universitário Mario Pontes Juca, Brasil E-mail: porangabarenata@gmail.com Tamires Santos Braga

ORCID: https://orcid.org/0000-0002-2969-1322 Centro Universitário Fametro, Brasil E-mail: tamiresbraga@hotmail.com

Graziela Santos da França

ORCID: https://orcid.org/0000-0001-8148-8359 Instituto Brasileiro de Medicina de Reabilitação, Brasil E-mail: grazielafranca25@gmail.com

Julio Cesar Pinheiro da Rocha Junior ORCID: https://orcid.org/0000-000-15475-6776 Instituto Brasileiro de Medicina de Reabilitação, Brasil E-mail: jcprjr@gmail.com

Maria Rosemary da Silva Gomes ORCID: https://orcid.org/0000-0002-8217-3647 Centro Universitário Santo Agostinho, Brasil

E-mail: mariarosemary2010@gmail.com

Sirlene Aparecida de Oliveira ORCID: https://orcid.org/0000-0003-2352-7983 Faculdade de Ensino Superior de Cacoal, Brasil E-mail: sirleneaparecidadeoliveira1988@gmail.com

Yesly Marinho da Rocha Barreto ORCID: https://orcid.org/0000-0002-5610-1420 Centro Universitário Brasileiro, Brasil E-mail: marinhoyesly@gmail.com

Ághata Monike Paula da Silva Lins ORCID: https://orcid.org/0000-0002-4606-8209 Centro Universitário Brasileiro, Brasil E-mail: aghatamonike@hotmail.com

Andreia Tomaz da Silva

ORCID: https://orcid.org/0000-0002-5262-2923 Faculdade Integrada Diferencial, Brasil E-mail: andreia_tomazz@yahoo.com.br

William França dos Santos ORCID: https://orcid.org/0000-0001-5750-7740

Universidade Federal de Pernambuco, Brasil E-mail: williamfds1997@gmail.com

Angelica Ribeiro do Nascimento Oliveira ORCID: https://orcid.org/0000-0002-7240-2004 Centro Universitário Maurício de Nassau, Brasil E-mail: angelicalribeiro.ar19@gmail.com

Ayrton Rogério Nascimento dos Santos ORCID: https://orcid.org/0000-0002-2518-5705

Faculdade Metropolitana de Manaus, Brasil E-mail: ayrtonn30@gmail.com 


\begin{abstract}
Resumo
Objetivo: Estudo bibliométrico que objetivou descrever as características da produção científica relacionada a feridas e estomias. Metodologia: Foram utilizados os termos ferimentos e lesões e estomia na coleta de dados que foi realizada em 06 de outubro de 2020, na base Web of Science (WoS). As variáveis observadas foram: ano de publicação, autores, país, idioma, instituições, revistas e áreas de pesquisa Resultados: Evidenciaram 52 indexações num período de vinte e sete anos (1993 a 2020). Das publicações 71\% foram artigos provenientes dos Estados Unidos da América (EUA), o Brasil não aparece no ranking das publicações, 100\% publicações foram na língua inglesa; todos foram artigos originais, o autor que mais publica encontra-se no EUA. Conclusão: Concluímos que a produção científica foi maior em 2016, o estudo também evidencia o envolvimento da enfermagem na temática, desde modo fica claro a necessidade de mais envolvimento científico da enfermagem brasileira com a temática para que haja um crescimento progressive sobre a temática no país.
\end{abstract}

Palavras-chave: Bibliometria; Ferimentos e lesões; Ostomia.

\begin{abstract}
Objective: Bibliometric study that aimed to describe the characteristics of scientific production related to wounds and ostomies. Methodology: The terms injuries and injuries and ostomy were used in the data collection that was carried out on October 6, 2020, in the Web of Science (WoS) database. The variables observed were: year of publication, authors, country, language, institutions, journals and research areas. Results: Evidenced 52 indexes in a period of twenty-seven years (1993 to 2020). Of the publications, 71\% were articles from the United States of America (USA), Brazil does not appear in the ranking of publications, $100 \%$ publications were in English; all were original articles, the author who publishes the most is in the USA. Conclusion: We conclude that the scientific production was higher in 2016, the study also shows the involvement of nursing in the subject, so it is clear the need for more scientific involvement of Brazilian nursing with the subject so that there is a progressive growth on the subject in the country.
\end{abstract}

Keywords: Bibliometry; Wounds and injuries; Ostomy.

\title{
Resumen
}

Objetivo: Estudio bibliométrico que tuvo como objetivo describir las características de la producción científica relacionada con heridas y ostomías. Metodología: Se utilizaron los términos lesiones y lesiones y ostomía en la recolección de datos que se realizó el 6 de octubre de 2020, en la base de datos de Web of Science (WoS). Las variables observadas fueron: año de publicación, autores, país, idioma, instituciones, revistas y áreas de investigación Resultados: Se evidenciaron 52 índices en un período de veintisiete años (1993 a 2020). De las publicaciones, el $71 \%$ fueron artículos de los Estados Unidos de América (EE. UU.), Brasil no aparece en el ranking de publicaciones, el $100 \%$ de las publicaciones fueron en inglés; todos fueron artículos originales, el autor que más publica está en USA. Conclusión: Se concluye que la producción científica fue mayor en 2016, el estudio también muestra el involucramiento de la enfermería en el tema, por lo que es clara la necesidad de un mayor involucramiento científico de la enfermería brasileña con el tema para que haya un crecimiento progresivo en el tema. en el país.

Palabras clave: Bibliometría; Heridas y traumatismos; Ostomía.

\section{Introdução}

Estomia, ostomia ou estoma são termos derivados do grego que significam "boca" ou "abertura" e indicam a exteriorização de qualquer víscera oca através do corpo, em outras palavras, é uma comunicação artificial entre os órgãos ou vísceras até o meio externo para a realização de drenagem, eliminação e/ou nutrição, que dependendo da localização anatômica assume nomes diferenciados como: colostomia, ileostomia e jejunostomia (Sampaio et al., 2008; Monge, 2008).

Dentre os tipos mais frequentes, tem-se a colostomia, caracterizada pela exteriorização do cólon por meio de um estoma através da parede abdominal, criando um novo trajeto para a saída do material fecal. No que diz respeito à epidemiologia das colostomias, dados do Departamento de Informática do Sistema Único de Saúde (DATASUS) revelam que no ano de 2017 foram realizadas no Brasil 6.347 colostomias, sendo o procedimento realizado majoritariamente na Região Sudeste (47,93\%), seguida do Nordeste (21\%), Sul (17,42\%), Sul (7,54\%) e Norte $(6,93 \%)$, com custo total de $\mathrm{R} \$$ 16.796.018,31 para o sistema de saúde brasileiro (Brasil, 2018). 
Vários fatores podem suscetibilizar o paciente a esse procedimento, dentre os quais podem-se citar: câncer de cólon e de reto, doença de Crohn, doença de Chagas, retocolite ulcerativa e ocorrência de traumas na região abdominal. Destaca-se que a presença da colostomia traz ao paciente uma experiência relacionada a restrições e mudanças de hábitos de vida (Monge, 2008), de forma que há limitações na movimentação corporal devido ao risco de descolamento da bolsa (Bellato et al., 2007; Stum et al., 2008).

Muitas das necessidades humanas básicas são alteradas em pacientes cirúrgicos, independente da cirurgia realizada. No contexto dos pacientes ostomizados, há cuidados específicos necessários para atender às demandas e às necessidades humanas básicas alteradas (Potter, 2006). Assim, para o cuidado de enfermagem, é necessário que a assistência seja direcionada às demandas específicas apresentadas pelo paciente, abordando aspectos holísticos do cuidar (de Souza Santos et al., 2006).

As feridas causadas pelas ostomias acabam influenciando na qualidade de vida do paciente, tendo em vista a hipervalorização da estética no mundo que vivemos, isto mantem o paciente longe de comemorações, encontros e festas, pois o mesmo acaba não se sentindo a vontade de estar nestes ambientes (Lara et al., 2011). Bem como as alterações na pele causadas pela lesão pode gerar quadros infecciosos, exudatos de odor fetido, e se não houver tratamento adequdaod pode evoluir para casos mais graves (Lara et al., 2011).

Diante da complexidade da ostomia e da importância dos cuidados de enfermagem ao paciente ostomizado, é essencial a realização de estudos que revelem quais são os cuidados da enfermagem ao paciente submetido a este procedimento, uma vez que tal compreensão é capaz de aprimorar o cuidado e qualificar a assistência a partir de evidências cientificamente embasadas. Frente ao exposto, este estudo tem como objetivo realizer uma bibliometria acerca de ostomias e feridas.

\section{Metodologia}

O estudo consiste em uma revisão bibliometrica, que consiste num método de análise quantitativa das produções científicas sobre um determinado assunto. Os estudos bibliométricos quantificam, descrevem e prognosticam o processo de comunicação escrita (Guedes \& Borschiver, 2005)

A base indexadora escolhida para a busca foi a Web of Science (WoS), que é uma base de dados que indexa produções científicas, multidisciplinares que reúnem um vasto conjuntos de editores e revistas que possuem um ponto de acesso global a grande parte da literatura científica nacional e internacional, oferece o acesso a teses, dissertações, periódicos, resumos, contendo mais de 38 mil publicações e atualizado semanalmente (Capes, 2017).

O período assumido para a busca foi 1993 a 2020, é a busca foi realizada no dia 06 de outubro de 2020 utilizando a Plataforma Capes na intranet da Universidade Federal da Bahia. A definição dos termos apropriados para busca ocorreu através da utilização do Descritores de Ciências da Saúde (Decs), de forma que foram elegidos: wounds and injuries (ferimentos e lesões) e ostomy (estomia).

Dentre os documentos disponíveis na WoS elegemos para esse estudo artigos originais publicados em periódicos. De forma que a amostra final foi composta por 52 artigos, após a busca combinada dos Decs com o operador booleando AND.

A coleta de dados foi realizada em adotando os descritores elegidos associado ao corte de temporal adotados. O resultado da busca retornou 52 artigos originais, dos quais foram extraídos os relatórios disponibilizados pela WoS.

Foram incluidos todos os artigos encontrados na base de dados independente de idioma, ano e tipo de estudo, foram excluidos todos os artigos repetidos na base de dados. Os indicadores adotados para a análise bibliométrica foram: ano, instituição, autores, países, agências financiadoras, idiomas, revistas/periódicos e área de pesquisa, seguido dos seus 
respectivos dados em números absolutos. Os dados foram demonstrados através de tabelas e gráficos adequados ao tipos de análises empreendida.

Os dados foram exportados e tratados no Excel®, versão 2016, através da construção de tabelas e gráficos, que permitiram a análise da progressão, bem como de cada variável detalhada. Por tratar-se de um estudo documental de cunho secundário de banco de dados de acesso aberto e não tratar-se de pesquisa com seres humanos dispensa apreciação de comitê de ética. Todavia, as autoras comprometem-se a citar todas as fontes utilizadas na construção desse documento.

\section{Resultados e Discussão}

Aplicada a estratégia de busca descrita na metodologia, retornaram inicialmente 52 artigos originais na WoS publicados no período de 1993 a 2020. Entre 1993-1995, 2002-2006, 2009-2011 constatou-se um hiato na produção de artigos sobre a temática. De forma que, primeira publicação sobre o tema de forma que não houve publicações neste periodo como mostra o Gráfico 1.

Gráfico 1. Número de publicações por ano de 1993-2020 utilizando os descritores wounds and injuries and ostomy. Brasil. 2021.

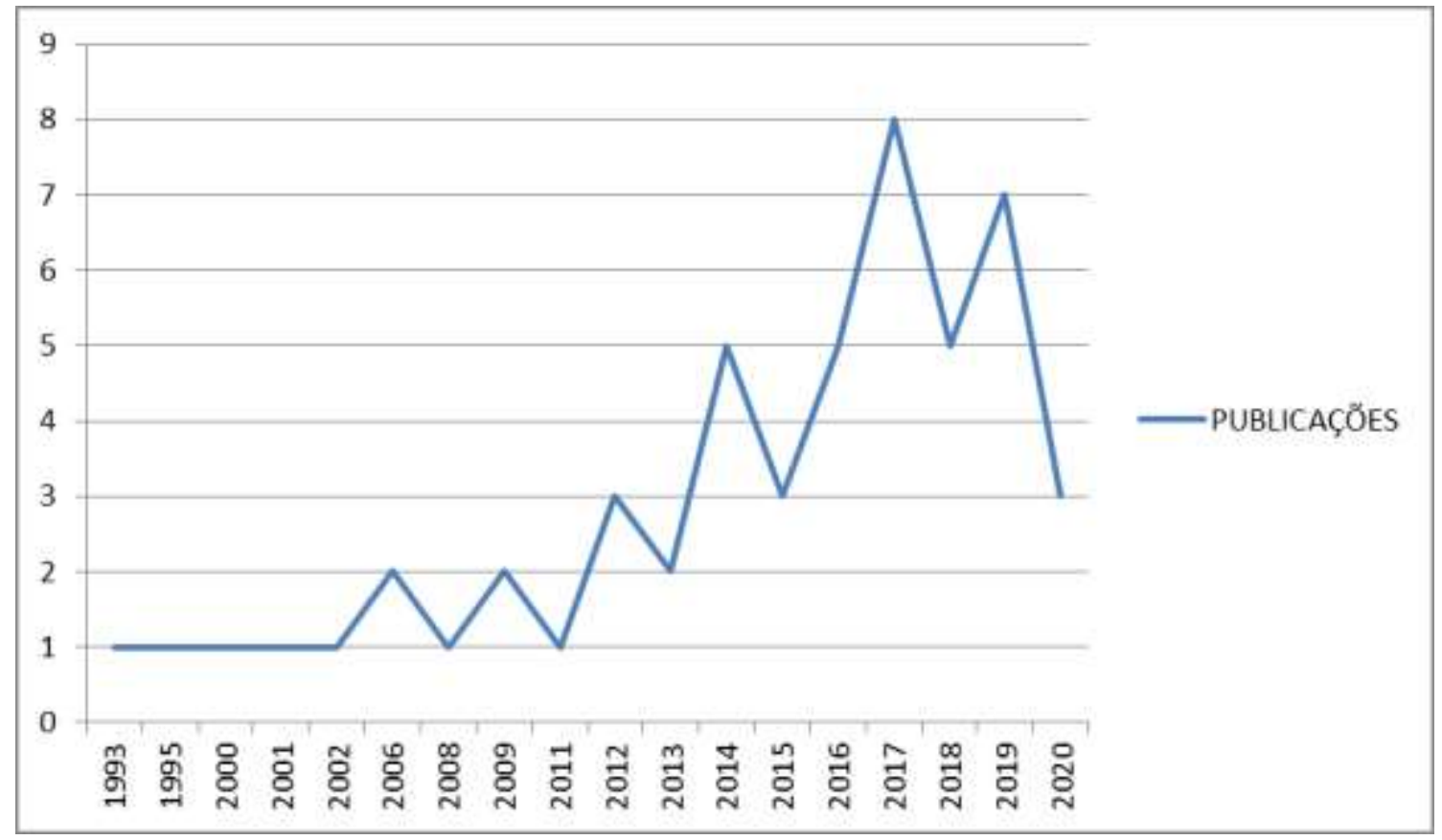

Fonte: Autores (2021).

Ao observar as 79 instituições que os autores diziam estar vinculados foram relacionadas 218 com pesquisadores que estudam a temática, ao realizar o cálculo de média de publicações por instituição, o resultado foi 0,67 (tendo em vista que alguns pesquisadores possuiam mais de um vinculo institucional). As dez (10) instituições que mais publicam no mundo detém $27(50,94 \%)$ de artigos publicados, as mesmas encontram-se situadas nos Estados Unidos e Japão, as seguintes informações encontram-se descritas na tabela a seguir. 
Tabela 1. As dez instituições que mais publicam sobre o tema utilizando os descritores wounds and injuries and ostomy. Brasil. 2021.

\begin{tabular}{l|c|c}
\hline Nome da Instituição & $\mathbf{N}^{*}$ & \% \\
\hline Universidade da Virginia & 4 & 7,69 \\
Centro Medico Militar Nacional Walter Reed & 4 & 7,69 \\
Universidade Duke & 3 & 5,79 \\
Clinica Mayo & 3 & 5,79 \\
Universidade de Medicina da Carolina do Sul & 3 & 5,79 \\
Universidade de Kanazawa & 2 & 3,84 \\
Centro Medico Militar San Antonio & 2 & 3,84 \\
Universidade de Sapporo City & 2 & 3,84 \\
Departamento de Defesa dos Estados Unidos & 2 & 3,84 \\
Universidade da Carolina do Norte & 2 & 3,84 \\
\hline
\end{tabular}

Fonte: Autores (2021).

Os autores que produzem sobre o tema no período estudado foi de 218 sendo GRAY, M. é o autor que mais produz sobre o tema com 4 artigos publicados sobre o tema, seguido de DUNCAN, JE. com 3 publicações. Com essas informações podemos afirmar que os maiores pesquisadores, produtores, autores sobre o tema estudado encontram-se nos Estados Unidos, como demonstra a Tabela 2.

Tabela 2. Os autores que mais publicam sobre o tema utilizando os descritores wounds and injuries and ostomy*. Brasil. 2021.

\begin{tabular}{l|c|c}
\hline Autor & $\mathbf{N}^{*}$ & \% \\
\hline GRAY, M. & 4 & 7,69 \\
DUNCAN, JE. & 3 & 5,76 \\
BONHAM, P & 2 & 3,84 \\
GLASGOW, SC. & 2 & 3,84 \\
KAITANI, T. & 2 & 3,84 \\
MCNICHOL, L. & 2 & 3,84 \\
NAKAGAMI, G. & 2 & 3,84 \\
RATLIFF, CR. & 2 & 3,84 \\
SANADA, H. & 2 & 3,84 \\
SUGAMA, J. & 2 & 3,84 \\
TAKEMURA, Y. & 2 & 3,84 \\
\hline
\end{tabular}

* Só foram incluídos autores com no mínimo 2 publicações Fonte: Autores (2021).

Em relação ao autores, o primeiro que mais publicam mundial sobre o tema é Mikel Gray é pós doutor e professor de enfermagem urologica da Universidade da Virginia, situada no estado da Virginia nos EUA, o mesmo dedica-se a pesquisa de vias urinarias, incontinência urinária, terapia de bio-feedback, bexiga neurogênica, dano cutâneo associado à umidade, dermatite associada à incontinência.

A terceira autora que mais publica é Phyllis Bonham e pós doutora em enfermagem com enfase em feridas, pela Universidade de Medicina da Carolina do Sul, localizada na Carolina do Sul nos EUA, a mesma dedica-se a pesquisas na área de enfermagem de Cuidados de Feridas, Testes vasculares não invasivos de extremidades inferiores (ABI, TBI, PPG) usando dispositivos portáteis, Diretrizes clínicas de cuidados de feridas baseadas em evidências. 
A origem dos autores registrou 92 países/territórios diferentes, não houve publicação de autores do Brasil. Os três primeiros países com autores que publicam sobre o tema estão os Estados Unidos, o Japão e a Turquia 83\% do autores total de produções mundiais, como mostra a tabela abaixo.

Tabela 3. Os países que mais publicam sobre o tema utilizando os descritores wounds and injuries and ostomy*. Brasil. 2021.

\begin{tabular}{l|c|c}
\hline PAIS & $\mathbf{N *}$ & \% \\
\hline Estados Unidos & 37 & 71,15 \\
Japão & 4 & 7,69 \\
Turquia & 3 & 5,76 \\
Canadá & 2 & 3,84 \\
China & 2 & 3,84 \\
Inglaterra & 1 & 1,92 \\
Índia & 1 & 1,92 \\
Israel & 1 & 1,92 \\
\hline
\end{tabular}

Fonte: Autores (2021).

Em relação ao países, os Estados Unidos ocupa o primeiro lugar no ranking de publicações, isso se confirmar por ser o país pioneiro em busca de feridas e ostomias, um dado que corrobora essa informação é o fato das universidades que mais publicam no mundo serem situadas lá, demonstra um grande interesse em aprimorar, aperfeiçoar os serviços e diminuir custos, como base nos dados acima os artigos o idioma predominante nos artigos é o inglês. O idioma adotado para a escrita dos artigos foi o inglês em 52 (100\% publicações.

No período estudado foram encontradas na base de dados 25 revistas que publicaram artigos originais sobre ostomia e feridas, média de 2 publicações por periódico. Dessas 3 (12\%) estão relacionadas a enfermagem, com um total de 16 (30,18\%) publicações sobre o tema estudado nessas revistas, representando uma por revista, sendo que tem mais publicações no ranking geral foi Journal of Wound Ostomy and Continence Nursing, conforme a tabela a seguir apresenta.

Tabela 4. Os períodicos que mais publicam sobre o tema utilizando os descritores wounds and injuries and ostomy*. Brasil. 2021.

\begin{tabular}{l|c|c}
\hline \multicolumn{1}{c|}{ PERÍODICOS } & N* & $\%$ \\
\hline Journal of Wound Ostomy and Continence Nursing & 14 & 26,92 \\
Journal of Trauma Injury Infection and Critical Care & 4 & 7,69 \\
American Suregon & 3 & 5,76 \\
Journal of Trauma and Acute Care Surgery & 3 & 5,76 \\
Diseases of The Colon Rectum & 2 & 3,84 \\
Military Medicine & 2 & 3,84 \\
Ostomy Wound Management & 2 & 3,84 \\
Advances in Neonatal Care & 1 & 1,92 \\
American Journal of Surgery & 1 & 1,92 \\
Archives of Surgery & 1 & 1,92 \\
Chinese Journal of Traumatology & 1 & 1,92
\end{tabular}




\begin{tabular}{l|c|c} 
Chronic Wound Care Management and Research & 1 & 1,92 \\
Clinics in Colon and Rectal Surgery & 1 & 1,92 \\
Critical Care Nurse & 1 & 1,92 \\
European Journal of Trauma and Emergency Surgery & 1 & 1,92 \\
Indian Journal of Palliative Care & 1 & 1,92 \\
International Journal of Ophthalmology & 1 & 1,92 \\
International Journal of Surgery Case Reports & 1 & 1,92 \\
International Wound Journal & 1 & 1,92 \\
Journal of Surgical Research & 1 & 1,92 \\
Journal of The American College of Surgeons & 1 & 1,92 \\
Journal of Vascular Nursing & 1 & 1,92 \\
Neonatal Network & 1 & 1,92 \\
Neuromodulation & 1 & 1,92 \\
\hline
\end{tabular}

Fonte: Autores (2021).

Nas revistas que mais publicam a Journal of Wound Ostomy and Continence Nursing ocupou o primeiro lugar com mais publicações, trata-se de uma revista, em Nova Jersey situada nos EUA, publica assuntos relacionados a ostomias e feridas, tem por objetivo atingir o público de enfermagem.

Em segundo lugar a revista Journal of Trauma Injury Infection and Critical Care, trata-se de uma revista de acesso aberto na qual retrata lesões traumáticas, com enfasê em aplicações clínicas, técnicas e novos desenvolvimentos no atendimento ao trauma, é uma revista multidisciplinar dos Estados Unidos.

Dentre as 15 áreas de conhecimento definidas pela WoS foram atribuídas aos artigos, podendo o mesmo receber mais de uma classificação (média de 1,37). As classificações atribuídas foram Cirurgia (Surgery) com 24 artigos (46,15\%), Enfermagem (NURSING) com 18 artigos (34,61\%), Medicina Interna Geral (GENERAL INTERNAL MEDICINE) com 10 artigos (19,23\%). Todos os artigos incluidos estão abaixo na Tabela 5.

Tabela 5. Todos os artigos incluidos na busca utilizando os descritores wounds and injuries and ostomy. Brasil. 2021.

\begin{tabular}{|c|c|c|c|}
\hline Autor & Ano & Titulo & Periódico \\
\hline Arnold, M; Yanez, C; Yanez, B & 2020 & $\begin{array}{l}\text { Wound Healing in the Long-Term Acute Care } \\
\text { Setting Using an Air Fluidized } \\
\text { Therapy/Continuous Low-Pressure Therapeutic } \\
\text { Bed A Multiple Case Series }\end{array}$ & $\begin{array}{l}\text { Journal of Wound Ostomy } \\
\text { And Continence Nursing }\end{array}$ \\
\hline $\begin{array}{l}\text { Cheng, V; Schellenberg, M; Inaba, K; } \\
\text { Matsushima, K; Warriner, Z; Trust, MD; } \\
\text { Lam, L; Demetriades, D }\end{array}$ & 2020 & $\begin{array}{l}\text { Contemporary Trends and Outcomes of Blunt } \\
\text { Traumatic Colon Injuries Requiring Resection }\end{array}$ & $\begin{array}{l}\text { Journal of Surgical } \\
\text { Research }\end{array}$ \\
\hline Gray, M & 2020 & $\begin{array}{l}\text { Context for Practice Wound Treatment Associate } \\
\text { Education Program, Medical Device-Related } \\
\text { Pressure Injuries, Fecal Ostomies, Female } \\
\text { External Collection Device, and Diabetic Foot } \\
\text { Ulcers }\end{array}$ & $\begin{array}{l}\text { Journal of Wound Ostomy } \\
\text { and Continence Nursing }\end{array}$ \\
\hline
\end{tabular}




\begin{tabular}{|c|c|c|c|}
\hline Schroeder, J; Sitzer, V & 2019 & $\begin{array}{l}\text { Nursing Care Guidelines for Reducing Hospital- } \\
\text { Acquired Nasogastric Tube-Related Pressure } \\
\text { Injuries }\end{array}$ & Critical Care Nurse \\
\hline Gray, M & 2019 & $\begin{array}{l}\text { Context for Practice: Technology and Wound } \\
\text { Care, Friction Skin Injuries, Financial Impact of } \\
\text { Living With an Ostomy, Lived Experiences of } \\
\text { Intermittent Catheterization, Diabetic Foot Ulcers }\end{array}$ & $\begin{array}{l}\text { Journal of Wound Ostomy } \\
\text { and Continence Nursing }\end{array}$ \\
\hline Scheans, P; Ecklund, M; Hampton, R & 2019 & $\begin{array}{l}\text { Developing Skin, Wound, and Ostomy Products } \\
\text { Formulary for a NICU in a Children's Hospital }\end{array}$ & Neonatal Network \\
\hline $\begin{array}{l}\text { Kumar, V; Kumawat, N; Thomas, JM; } \\
\text { Kumar, A; Sharma, A; Kamal, M }\end{array}$ & 2019 & $\begin{array}{l}\text { Challenges Encountered in Placement of Ostomy } \\
\text { Bag for Palliative Care in a Patient with Colorectal } \\
\text { Cancer }\end{array}$ & $\begin{array}{l}\text { Indian Journal of Palliative } \\
\text { Care }\end{array}$ \\
\hline Huang, GB; Hu, P; Gao, JM; Lin, X & 2019 & $\begin{array}{l}\text { Analysis of early treatment of multiple injuries } \\
\text { combined with severe pelvic fracture }\end{array}$ & $\begin{array}{l}\text { Chinese Journal of } \\
\text { Traumatology }\end{array}$ \\
\hline $\begin{array}{l}\text { Manley, NR; Sharpe, JP; Lewis, RH; } \\
\text { Iltis, MS; Chaudhuri, R; Fabian, TC; } \\
\text { Croce, MA; Magnotti, LJ }\end{array}$ & 2019 & $\begin{array}{l}\text { Analysis of over } 2 \text { decades of colon injuries } \\
\text { identifies optimal method of diversion: Does an } \\
\text { end justify the means? }\end{array}$ & $\begin{array}{l}\text { Journal of Trauma and } \\
\text { Acute Care Surgery }\end{array}$ \\
\hline $\begin{array}{l}\text { Suzuki, M; Matsushima, H; Uehara, K; } \\
\text { Saiki, T; Hayamizu, A; Kamisasanuki, T; } \\
\text { Sugiki, D }\end{array}$ & 2019 & $\begin{array}{l}\text { Stomal stenosis during gradual closure of a } \\
\text { traumatic abdominal wall hernia }\end{array}$ & $\begin{array}{l}\text { International Journal of } \\
\text { Surgery Case Reports }\end{array}$ \\
\hline $\begin{array}{l}\text { Bliss, DZ; Gurvich, OV; Hurlow, J; } \\
\text { Cefalu, JE; Gannon, A; Wilhems, A; } \\
\text { Wiltzen, KR; Gannon, E; Lee, H; } \\
\text { Borchert, K; Trammel, SH }\end{array}$ & 2018 & $\begin{array}{l}\text { Evaluation of Validity and Reliability of a Revised } \\
\text { Incontinence-Associated Skin Damage Severity } \\
\text { Instrument (IASDD2) by } 3 \text { Groups of Nursing } \\
\text { Staff }\end{array}$ & $\begin{array}{l}\text { Journal of Wound Ostomy } \\
\text { and Continence Nursing }\end{array}$ \\
\hline $\begin{array}{l}\text { Johnston, LR; Bradley, MJ; Rodriguez, } \\
\text { CJ; McNally, MP; Elster, EA; Duncan, } \\
\text { JE }\end{array}$ & 2018 & $\begin{array}{l}\text { Assessing Risk and Related Complications after } \\
\text { Reversal of Combat-Associated Ostomies }\end{array}$ & $\begin{array}{l}\text { Journal of the American } \\
\text { College of Surgeons }\end{array}$ \\
\hline Hill, R; Rennie, MY; Douglas, J & 2018 & $\begin{array}{l}\text { Using Bacterial Fluorescence Imaging and } \\
\text { Antimicrobial Stewardship to Guide Wound } \\
\text { Management Practices: A Case Series }\end{array}$ & $\begin{array}{l}\text { Ostomy Wound } \\
\text { Management }\end{array}$ \\
\hline Wong, VK & 2018 & $\begin{array}{l}\text { Establishing a Hybrid Wound and Ostomy } \\
\text { Continuing Education Program }\end{array}$ & $\begin{array}{l}\text { Nursing } \quad \text { Education } \\
\text { Perspectives }\end{array}$ \\
\hline Yamamoto, R; Logue, AJ; Muir, MT & 2018 & Colon Trauma: Evidence-Based Practices & $\begin{array}{l}\text { Clinics In Colon And Rectal } \\
\text { Surgery }\end{array}$ \\
\hline $\begin{array}{l}\text { Schmitt, S; Andries, MK; Ashmore, PM; } \\
\text { Brunette, G; Judge, K; Bonham, PA }\end{array}$ & 2017 & $\begin{array}{l}\text { WOCN Society Position Paper Avoidable Versus } \\
\text { Unavoidable Pressure Ulcers/Injuries }\end{array}$ & $\begin{array}{l}\text { Journal of Wound Ostomy } \\
\text { and Continence Nursing }\end{array}$ \\
\hline Famorca, M; Beauchaine, D; Angulo, N & 2017 & $\begin{array}{l}\text { Management of a Complex Peristomal } \\
\text { Calciphylaxis A Case Study }\end{array}$ & $\begin{array}{l}\text { Journal of Wound Ostomy } \\
\text { and Continence Nursing }\end{array}$ \\
\hline $\begin{array}{l}\text { Rosen, DR; Hwang, GS; Ault, GT; } \\
\text { Ortega, AE; Cologne, KG }\end{array}$ & 2017 & $\begin{array}{l}\text { Operative management of diverticulitis in a } \\
\text { tertiary care center }\end{array}$ & $\begin{array}{l}\text { American Journal of } \\
\text { Surgery }\end{array}$ \\
\hline Ratliff, CR & 2017 & $\begin{array}{l}\text { Descriptive study of the frequency of medical } \\
\text { adhesive-related skin injuries in a vascular clinic }\end{array}$ & $\begin{array}{l}\text { Journal of Vascular } \\
\text { Nursing }\end{array}$ \\
\hline Boyle, DK; Bergquist-Beringer, S; & 2017 & Relationship of Wound, Ostomy, and Continence & Journal of Wound Ostomy \\
\hline
\end{tabular}




\begin{tabular}{|c|c|c|c|}
\hline Cramer, E & & $\begin{array}{l}\text { Certified Nurses and Healthcare-Acquired } \\
\text { Conditions in Acute Care Hospitals }\end{array}$ & and Continence Nursing \\
\hline $\begin{array}{l}\text { Ratliff, CR; Droste, LR; Bonham, P; } \\
\text { Crestodina, L; Johnson, JJ; Kelechi, T; } \\
\text { Varnado, MF; Palmer, R }\end{array}$ & 2017 & $\begin{array}{l}\text { WOCN } 2016 \text { Guideline for Prevention and } \\
\text { Management of Pressure Injuries (Ulcers) An } \\
\text { Executive Summary }\end{array}$ & $\begin{array}{l}\text { Journal of Wound Ostomy } \\
\text { and Continence Nursing }\end{array}$ \\
\hline Kucera, WB; Jezior, JR; Duncan, JE & 2017 & $\begin{array}{l}\text { Management of Post-Traumatic } \\
\text { Rectovesical/Rectourethral Fistulas: Case Series } \\
\text { of Complicated Injuries in Wounded Warriors and } \\
\text { Review of the Literature }\end{array}$ & Military Medicine \\
\hline $\begin{array}{l}\text { Yates, S; McNichol, L; Heinecke, SB; } \\
\text { Gray, M }\end{array}$ & 2017 & $\begin{array}{l}\text { Embracing the Concept, Defining the Practice, and } \\
\text { Changing the Outcome: Setting the Standard for } \\
\text { Medical Adhesive-Related Skin Injury } \\
\text { Interventions in WOC Nursing Practice }\end{array}$ & $\begin{array}{l}\text { Journal of Wound Ostomy } \\
\text { and Continence Nursing }\end{array}$ \\
\hline $\begin{array}{l}\text { Nist, MD; Rodgers, EA; Ruth, BM; } \\
\text { Bertoni, CB; Bartman, T; Keller, LA; } \\
\text { Dail, JW; Gardikes-Gingery, R; Sheperd, } \\
\text { EG }\end{array}$ & 2016 & $\begin{array}{l}\text { Skin Rounds A Quality Improvement Approach to } \\
\text { Enhance Skin Care in the Neonatal Intensive Care } \\
\text { Unit }\end{array}$ & Advances in Neonatal Care \\
\hline $\begin{array}{l}\text { Shah, M; Ellis, CT; Phillips, MR; } \\
\text { Marzinsky, A; Adamson, W; Weiner, T; } \\
\text { Erickson, K; Lee, S; Lange, PA; McLean, } \\
\text { SE }\end{array}$ & 2016 & $\begin{array}{l}\text { Preoperative Bowel Preparation before Elective } \\
\text { Bowel Resection or Ostomy Closure in the } \\
\text { Pediatric Patient Population Has No Impact on } \\
\text { Outcomes: A Prospective Randomized Study }\end{array}$ & American Surgeon \\
\hline $\begin{array}{l}\text { Narang, } \quad \mathrm{S} ; \quad \text { Srinivasan, } \quad \text { SK; } \\
\text { Zinboonyahgoon, N; Sampson, CE }\end{array}$ & 2016 & $\begin{array}{l}\text { Upper Antero-Medial Thigh as an Alternative Site } \\
\text { for Implantation of Intrathecal Pumps: A Case } \\
\text { Series }\end{array}$ & Neuromodulation \\
\hline $\begin{array}{l}\text { Alp, MN; Oken, OF; Sargon, MF; } \\
\text { Ucaner, A }\end{array}$ & 2016 & $\begin{array}{l}\text { Histopathologic effects of a low molecular weight } \\
\text { heparin on bone healing in rats: a promising } \\
\text { adjuvant in dacryocystorhinostomy }\end{array}$ & $\begin{array}{l}\text { International Journal of } \\
\text { Ophthalmology }\end{array}$ \\
\hline $\begin{array}{l}\text { Lachenbruch, C; Ribble, D; Emmons, K; } \\
\text { VanGilder, C }\end{array}$ & 2016 & $\begin{array}{l}\text { Pressure Ulcer Risk in the Incontinent Patient } \\
\text { Analysis of Incontinence and Hospital-Acquired } \\
\text { Pressure Ulcers From the International Pressure } \\
\text { Ulcer Prevalence (TM) Survey }\end{array}$ & $\begin{array}{l}\text { Journal of Wound Ostomy } \\
\text { and Continence Nursing }\end{array}$ \\
\hline $\begin{array}{l}\text { Kaitani, T; Nakagami, G; Iizaka, S; } \\
\text { Fukuda, T; Oe, M; Igarashi, A; Mori, T; } \\
\text { Takemura, Y; Mizokami, Y; Sugama, J; } \\
\text { Sanada, H }\end{array}$ & 2015 & $\begin{array}{l}\text { Cost-utility analysis of an advanced pressure ulcer } \\
\text { management protocol followed by trained wound, } \\
\text { ostomy, and continence nurses }\end{array}$ & $\begin{array}{l}\text { Wound Repair and } \\
\text { Regeneration }\end{array}$ \\
\hline $\begin{array}{l}\text { McNichol, L; Watts, C; Mackey, D; } \\
\text { Beitz, JM; Gray, M }\end{array}$ & 2015 & $\begin{array}{l}\text { Identifying the Right Surface for the Right Patient } \\
\text { at the Right Time: Generation and Content } \\
\text { Validation of an Algorithm for Support Surface } \\
\text { Selection }\end{array}$ & $\begin{array}{l}\text { Journal of Wound Ostomy } \\
\text { and Continence Nursing }\end{array}$ \\
\hline $\begin{array}{l}\text { Kaitani, T; Nakagami, G; Sugama, J; } \\
\text { Tachi, M; Matsuyama, Y; Miyachi, Y; } \\
\text { Nagase, T; Takemura, Y; Sanada, H }\end{array}$ & 2015 & $\begin{array}{l}\text { Evaluation of an advanced pressure ulcer } \\
\text { management protocol followed by trained wound, } \\
\text { ostomy, and continence nurses: a non-randomized } \\
\text { controlled trial }\end{array}$ & $\begin{array}{l}\text { Chronic Wound Care } \\
\text { Management And Research }\end{array}$ \\
\hline Sullivan, $\mathrm{R}$ & 2014 & A 5-Year Retrospective Study of Descriptors & Wounds-A Compendium of \\
\hline
\end{tabular}




\begin{tabular}{|c|c|c|c|}
\hline & & $\begin{array}{l}\text { Associated With Identification of Stage I and } \\
\text { Suspected Deep Tissue Pressure Ulcers in Persons } \\
\text { with Darkly Pigmented Skin }\end{array}$ & $\begin{array}{lll}\text { Clinical } & \text { Research and } \\
\text { Practice } & & \\
\end{array}$ \\
\hline $\begin{array}{l}\text { Merchant, N; Boudana, D; Willoughby, } \\
\text { L; Lin, JJ; Rehou, S; Shahrokhi, S; } \\
\text { Jeschke, MG }\end{array}$ & 2014 & $\begin{array}{l}\text { Management of adult patients with buttock and } \\
\text { perineal burns: The Ross Tilley Burn Centre } \\
\text { experience }\end{array}$ & $\begin{array}{l}\text { Journal of Trauma and } \\
\text { Acute Care Surgery }\end{array}$ \\
\hline $\begin{array}{l}\text { Hanna, JM; Turley, R; Castleberry, A; } \\
\text { Hopkins, T; Peterson, AC; Mantyh, C; } \\
\text { Migaly, J }\end{array}$ & 2014 & $\begin{array}{l}\text { Surgical Management of Complex Rectourethral } \\
\text { Fistulas in Irradiated and Nonirradiated Patients }\end{array}$ & $\begin{array}{l}\text { Diseases of the Colon \& } \\
\text { Rectum }\end{array}$ \\
\hline $\begin{array}{l}\text { Glasgow, SC; Heafner, TA; Watson, } \\
\text { JDB; Aden, JK; Perry, WB }\end{array}$ & 2014 & $\begin{array}{l}\text { Initial Management and Outcome of Modern } \\
\text { Battlefield Anal Trauma }\end{array}$ & $\begin{array}{l}\text { Diseases of the Colon \& } \\
\text { Rectum }\end{array}$ \\
\hline $\begin{array}{l}\text { Mackintosh, R; Gwilliam, A; Williams, } \\
\text { M }\end{array}$ & 2014 & Teaching the Fruits of Pressure Ulcer Staging & $\begin{array}{l}\text { Journal Of Wound Ostomy } \\
\text { and Continence Nursing }\end{array}$ \\
\hline Yang, F & 2013 & $\begin{array}{l}\text { Use of Polypropylene Mesh in the Management of } \\
\text { a Contaminated Large Ventral Hernia: a } \\
\text { Contraindication or a Solution? }\end{array}$ & American Surgeon \\
\hline $\begin{array}{l}\text { Brown, SR; Swisher, JP; Hofmann, LJ; } \\
\text { Coviello, LC; Davis, KG }\end{array}$ & 2013 & $\begin{array}{l}\text { Surgical Management and Associated } \\
\text { Complications of Penetrating Rectal Injuries } \\
\text { Sustained in Iraq and Afghanistan }\end{array}$ & Military Medicine \\
\hline $\begin{array}{l}\text { Glasgow, SC; Steele, SR; Duncan, JE; } \\
\text { Rasmussen, TE }\end{array}$ & 2012 & $\begin{array}{l}\text { Epidemiology of modern battlefield colorectal } \\
\text { trauma: A review of } 977 \text { coalition casualties }\end{array}$ & $\begin{array}{l}\text { Journal of Trauma and } \\
\text { Acute Care Surgery }\end{array}$ \\
\hline $\begin{array}{l}\text { Gumus, M; Boyuk, A; Kapan, M; Onder, } \\
\text { A; Taskesen, F; Aliosmanoglu, I; Tufek, } \\
\text { A; Aldemir, M }\end{array}$ & 2012 & $\begin{array}{l}\text { Unusual extraperitoneal rectal injuries: a } \\
\text { retrospective study }\end{array}$ & $\begin{array}{l}\text { European Journal of } \\
\text { Trauma and Emergency } \\
\text { Surgery }\end{array}$ \\
\hline Sato, T; Ichioka, S & 2012 & $\begin{array}{l}\text { Pressure Ulcer Occurrence Following the Great } \\
\text { East Japan Earthquake: Observations from a } \\
\text { Disaster Medical Assistance Team }\end{array}$ & $\begin{array}{l}\text { Ostomy } \quad \text { Wound } \\
\text { Management }\end{array}$ \\
\hline $\begin{array}{l}\text { Ozer, MT; Coskun, AK; Ozerhan, IH; } \\
\text { Ersoz, N; Yildiz, R; Sinan, H; Demirbas, } \\
\text { S; Kozak, O; Uzar, AI; Cetiner, S }\end{array}$ & 2011 & $\begin{array}{l}\text { Use of vacuum-assisted closure (VAC (TM)) in } \\
\text { high-energy complicated perineal injuries: } \\
\text { analysis of nine cases }\end{array}$ & $\begin{array}{l}\text { International Wound } \\
\text { Journal }\end{array}$ \\
\hline $\begin{array}{l}\text { Rosenthal, LHS; Benninger, MS; Stone, } \\
\mathrm{CH} \text {; Zacharek, MA }\end{array}$ & 2009 & $\begin{array}{l}\text { Wound healing in the rabbit paranasal sinuses } \\
\text { after Coblation: Evaluation for use in endoscopic } \\
\text { sinus surgery }\end{array}$ & $\begin{array}{l}\text { American Journal of } \\
\text { Rhinology \& Allergy }\end{array}$ \\
\hline $\begin{array}{l}\text { Vertrees, A; Wakefield, M; Pickett, C; } \\
\text { Greer, L; Wilson, A; Gillern, S; Nelson, } \\
\text { J; Aydelotte, J; Stojadinovic, A; Shriver, } \\
\text { C }\end{array}$ & 2009 & $\begin{array}{l}\text { Outcomes of Primary Repair and Primary } \\
\text { Anastomosis in War-Related Colon Injuries }\end{array}$ & $\begin{array}{l}\text { Journal of Trauma-Injury } \\
\text { Infection and Critical Care }\end{array}$ \\
\hline $\begin{array}{l}\text { Iqbal, CW; Cullinane, DC; Schiller, HJ; } \\
\text { Sawyer, MD; Zietlow, SP; Farley, DR }\end{array}$ & 2008 & $\begin{array}{l}\text { Surgical management and outcomes of } 165 \\
\text { colonoscopic perforations from a single institution }\end{array}$ & Archives of Surgery \\
\hline Steele, SE & 2006 & When trauma means a stoma & $\begin{array}{l}\text { Journal of Wound Ostomy } \\
\text { and Continence Nursing }\end{array}$ \\
\hline $\begin{array}{l}\text { Doughty, D; Ramundo, J; Bonham, P; } \\
\text { Beitz, J; Erwin-Toth, P; Anderson, R; }\end{array}$ & 2006 & Issues and challenges in staging of pressure ulcers & $\begin{array}{l}\text { Journal of Wound Ostomy } \\
\text { and Continence Nursing }\end{array}$ \\
\hline
\end{tabular}




\begin{tabular}{|c|c|c|c|}
\hline Rolstad, BS & & & \\
\hline Kelly, ME; Behrman, SW & 2002 & $\begin{array}{l}\text { The safety and efficacy of prosthetic hernia repair } \\
\text { in clean-contaminated and contaminated wounds }\end{array}$ & American Surgeon \\
\hline $\begin{array}{l}\text { Johnson, JW; Gracias, VH; Schwab, CW; } \\
\text { Reilly, PM; Kauder, DR; Shapiro, MB; } \\
\text { Dabrowski, GP; Rotondo, MF }\end{array}$ & 2001 & $\begin{array}{l}\text { Evolution in damage control for exsanguinating } \\
\text { penetrating abdominal injury }\end{array}$ & $\begin{array}{l}\text { Journal of Trauma-Injury } \\
\text { Infection and Critical Care }\end{array}$ \\
\hline $\begin{array}{l}\text { Dente, CJ; Tyburski, J; Wilson, RF; } \\
\text { Collinge, J; Steffes, C; Carlin, A }\end{array}$ & 2000 & $\begin{array}{l}\text { Ostomy as a risk factor for posttraumatic infection } \\
\text { in penetrating colonic injuries: Univariate and } \\
\text { multivariate analyses }\end{array}$ & $\begin{array}{l}\text { Journal of Trauma-Injury } \\
\text { Infection and Critical Care }\end{array}$ \\
\hline KLAUSNER, JM; ROZIN, RR & 1995 & $\begin{array}{l}\text { LATE ABDOMINAL COMPLICATIONS IN } \\
\text { WAR WOUNDED }\end{array}$ & $\begin{array}{l}\text { Journal of Trauma-Injury } \\
\text { Infection and Critical Care }\end{array}$ \\
\hline NICHOLS, RL; SMITH, JW & 1993 & $\begin{array}{l}\text { RISK OF INFECTION, INFECTING FLORA } \\
\text { AND TREATMENT CONSIDERATIONS IN } \\
\text { PENETRATING ABDOMINAL-TRAUMA }\end{array}$ & $\begin{array}{l}\text { Surgery Gynecology \& } \\
\text { Obstetrics }\end{array}$ \\
\hline Arnold, M; Yanez, C; Yanez, B & 2020 & $\begin{array}{l}\text { Wound Healing in the Long-Term Acute Care } \\
\text { Setting Using an Air Fluidized } \\
\text { Therapy/Continuous Low-Pressure Therapeutic } \\
\text { Bed A Multiple Case Series }\end{array}$ & $\begin{array}{l}\text { Journal of Wound Ostomy } \\
\text { and Continence Nursing }\end{array}$ \\
\hline $\begin{array}{l}\text { Cheng, V; Schellenberg, M; Inaba, K; } \\
\text { Matsushima, K; Warriner, Z; Trust, MD; } \\
\text { Lam, L; Demetriades, D }\end{array}$ & 2020 & $\begin{array}{l}\text { Contemporary Trends and Outcomes of Blunt } \\
\text { Traumatic Colon Injuries Requiring Resection }\end{array}$ & $\begin{array}{l}\text { Journal of } \quad \text { Surgical } \\
\text { Research }\end{array}$ \\
\hline Gray, M & 2020 & $\begin{array}{l}\text { Context for Practice Wound Treatment Associate } \\
\text { Education Program, Medical Device-Related } \\
\text { Pressure Injuries, Fecal Ostomies, Female } \\
\text { External Collection Device, and Diabetic Foot } \\
\text { Ulcers }\end{array}$ & $\begin{array}{l}\text { Journal of Wound Ostomy } \\
\text { and Continence Nursing }\end{array}$ \\
\hline Schroeder, J; Sitzer, V & 2019 & $\begin{array}{l}\text { Nursing Care Guidelines for Reducing Hospital- } \\
\text { Acquired Nasogastric Tube-Related Pressure } \\
\text { Injuries }\end{array}$ & Critical Care Nurse \\
\hline Gray, M & 2019 & $\begin{array}{l}\text { Context for Practice: Technology and Wound } \\
\text { Care, Friction Skin Injuries, Financial Impact of } \\
\text { Living With an Ostomy, Lived Experiences of } \\
\text { Intermittent Catheterization, Diabetic Foot Ulcers }\end{array}$ & $\begin{array}{l}\text { Journal of Wound Ostomy } \\
\text { and Continence Nursing }\end{array}$ \\
\hline Scheans, P; Ecklund, M; Hampton, R & 2019 & $\begin{array}{l}\text { Developing Skin, Wound, and Ostomy Products } \\
\text { Formulary for a NICU in a Children's Hospital }\end{array}$ & Neonatal Network \\
\hline $\begin{array}{l}\text { Kumar, V; Kumawat, N; Thomas, JM; } \\
\text { Kumar, A; Sharma, A; Kamal, M }\end{array}$ & 2019 & $\begin{array}{l}\text { Challenges Encountered in Placement of Ostomy } \\
\text { Bag for Palliative Care in a Patient with Colorectal } \\
\text { Cancer }\end{array}$ & $\begin{array}{l}\text { Indian Journal of Palliative } \\
\text { Care }\end{array}$ \\
\hline Huang, GB; Hu, P; Gao, JM; Lin, X & 2019 & $\begin{array}{l}\text { Analysis of early treatment of multiple injuries } \\
\text { combined with severe pelvic fracture }\end{array}$ & $\begin{array}{l}\text { Chinese Journal of } \\
\text { Traumatology }\end{array}$ \\
\hline $\begin{array}{l}\text { Manley, NR; Sharpe, JP; Lewis, RH; } \\
\text { Iltis, MS; Chaudhuri, R; Fabian, TC; } \\
\text { Croce, MA; Magnotti, LJ }\end{array}$ & 2019 & $\begin{array}{l}\text { Analysis of over } 2 \text { decades of colon injuries } \\
\text { identifies optimal method of diversion: Does an } \\
\text { end justify the means? }\end{array}$ & $\begin{array}{l}\text { Journal of Trauma and } \\
\text { Acute Care Surgery }\end{array}$ \\
\hline
\end{tabular}




\begin{tabular}{|c|c|c|c|}
\hline $\begin{array}{l}\text { Suzuki, M; Matsushima, H; Uehara, K; } \\
\text { Saiki, T; Hayamizu, A; Kamisasanuki, T; } \\
\text { Sugiki, D }\end{array}$ & 2019 & $\begin{array}{l}\text { Stomal stenosis during gradual closure of a } \\
\text { traumatic abdominal wall hernia }\end{array}$ & $\begin{array}{l}\text { International Journal of } \\
\text { Surgery Case Reports }\end{array}$ \\
\hline $\begin{array}{l}\text { Bliss, DZ; Gurvich, OV; Hurlow, J; } \\
\text { Cefalu, JE; Gannon, A; Wilhems, A; } \\
\text { Wiltzen, KR; Gannon, E; Lee, H; } \\
\text { Borchert, K; Trammel, SH }\end{array}$ & 2018 & $\begin{array}{l}\text { Evaluation of Validity and Reliability of a Revised } \\
\text { Incontinence-Associated Skin Damage Severity } \\
\text { Instrument (IASDD2) by } 3 \text { Groups of Nursing } \\
\text { Staff }\end{array}$ & $\begin{array}{l}\text { Journal of Wound Ostomy } \\
\text { and Continence Nursing }\end{array}$ \\
\hline $\begin{array}{l}\text { Johnston, LR; Bradley, MJ; Rodriguez, } \\
\text { CJ; McNally, MP; Elster, EA; Duncan, } \\
\text { JE }\end{array}$ & 2018 & $\begin{array}{l}\text { Assessing Risk and Related Complications after } \\
\text { Reversal of Combat-Associated Ostomies }\end{array}$ & $\begin{array}{l}\text { Journal of The American } \\
\text { College of Surgeons }\end{array}$ \\
\hline Hill, R; Rennie, MY; Douglas, J & 2018 & $\begin{array}{l}\text { Using Bacterial Fluorescence Imaging and } \\
\text { Antimicrobial Stewardship to Guide Wound } \\
\text { Management Practices: A Case Series }\end{array}$ & $\begin{array}{l}\text { Ostomy } \\
\text { Management }\end{array}$ \\
\hline
\end{tabular}

Fonte: Autores (2021).

\section{Considerações Finais}

A presente pesquisa atingiu o obejtivo que foi elencar a produção bibliométrica mundial de 1993-2020 de acordo com termos wounds and injuries (feridas e lesões) and ostomy (ostomias). Deve-se considerar a importância de pesquisas in loco, pois as mesmas possibilitam mostrar a realidade das instituições hospitalares permitindo o avanço das produções sobre tema bem como a criação de protocolos que visam diminuir o número de feridas em ostomias.

Constatou-se crescimento exponencial do número de artigos produzidos a partir de 2005, o ano de maior produção foi 2016, os autores e instituições destaques sobre a temáticaestão situados nos Estados Unidos e a língua de publicação foi o inglês. Os grandes produtores sobre a temática estão vinculados a instituições prementes no campo científicos e acabaram por executar atividades de coordenação e intervenção sobre a temática em pauta.

Destaca-se o crescimento de periódicos específicos sobre o tema, as enfermeiras estão ligadas não somente na execução prática dos cuidados, mas também na produção de conhecimento científico, que gera mudanças, melhorias e inovaçõesno âmbito da qualidade da assistência em saúde aos pacientes ostomizados., contudo notamos que o Brasil ainda tem muito o que avançar para tornar-seum país de primeiro mundo em relação ao referido tema, mas que já se encontra em busca desses avanços.

No entanto todo o tema é de extrema importância para toda a equipe multidisciplinar em saúde e gestores hospitalares, visto que o aprimoramento desse conhecimento e as práticas seguras levam a redução de feridas nas ostomies e reduzem os danos causados aos pacientes, a diminuição de tempo de internamento e a redução de custos hospitalares e a satifação do atendimento do cliente.

\section{Referências}

Bellato, R., Maruyama, S. A. T., Silva, C. M., \& Castro, P. (2007). A condição crônica ostomia e as repercussões que traz para a vida da pessoa e sua família. Ciência, Cuidado e Saúde, 6(1), 40-40.

CAPES. (2017). http://www.periodicos.capes.gov.br/

de Souza Santos, G., Leal, S. M. C., \& Vargas, M. A. (2006). Conhecendo as vivências de mulheres ostomizadas: contribuições para o planejamento do cuidado de enfermagem. Online Brazilian Journal of Nursing, 5(1), 27-37.

Guedes, V. L., \& Borschiver, S. (2005). Bibliometria: uma ferramenta estatística para a gestão da informação e do conhecimento, em sistemas de informação, de comunicação e de avaliação científica e tecnológica. Encontro Nacional de Ciência da Informação, 6(1), 18. 
Research, Society and Development, v. 10, n. 11, e166101118682, 2021

(CC BY 4.0) | ISSN 2525-3409 | DOI: http://dx.doi.org/10.33448/rsd-v10i11.18682

Lara, M. O., Júnior, A. D. C. P., de Figueiredo Pinto, J. S., Vieira, N. F., \& Wichr, P. (2011). Significado da ferida para portadores de úlceras crônicas. Cogitare Enfermagem, 16(3), 471-477.

Marinho, M. C. (2016). Assistência de enfermagem frente à pessoa ostomizada: revisão integrativa.

Monge, R. A., \& Avelar, M. D. C. Q. (2009). A assistência de enfermagem aos pacientes com estomia intestinal: percepção dos enfermeiros. Online braz. j. nurs.(Online).

Monteiro, A. K. D. C., Costa, C. P. V. D., Campos, M. D. O. B., \& Monteiro, A. K. D. C. (2016). Aplicabilidade da teoria de Callista Roy no cuidado de enfermagem ao estomizado. Rev. enferm. atenção saúde, 84-92.

Mota, M. S., \& Gomes, G. C. (2013). Mudanças no processo de viver do paciente estomizado após a cirurgia. Journal of Nursing UFPE/Revista de Enfermagem UFPE, 7(12).

Nascimento, C. D. M. D. S., Trindade, G. L. B., Luz, M. H. B. A., \& Santiago, R. F. (2011). Vivência do paciente estomizado: uma contribuição para a assistência de enfermagem. Texto \& Contexto-Enfermagem, 20, 557-564.

Pelazza, B. B., de Paula, C. R., Evangelista, R. A., de Assis Bueno, A., Trincaus, M. R., Leite, G. R., ... \& Claudio, R. H. A. (2016). Ações Curativas de Enfermeiros em Pessoas Estomizadas: Revisão Bibliográfica. OLive Revista Científica Eletrônica-ISSN 2448-4172, 1(1), 42-58.

Potter, P. A. (2006). Fundamentos de enfermagem. Elsevier Brasil.

Sampaio, F. A. A., Aquino, P. D. S., Araújo, T. L. D., \& Galvão, M. T. G. (2008). Assistência de enfermagem a paciente com colostomia: aplicação da teoria de Orem. Acta Paulista de Enfermagem, 21, 94-100.

Silva, E. S. D., Castro, D. S. D., Garcia, T. R., Romero, W. G., \& Primo, C. C. (2016). Tecnologia do cuidado à pessoa com colostomia: diagnósticos e intervenções de enfermagem. Revista Mineira de Enfermagem, 20.

Souza, N. Z., Calcagno, G. G., Xavier, D. M., Mota, M. S., Alvares, S. Q., \& Souza, J. L. (2012). O papel do enfermeiro no serviço de estomaterapia. II Jornada Internacional de Enfermagem Unifra, 2.

Stumm, E. M. F., Oliveira, E. R. A., \& Kirschner, R. M. (2008). Perfil de pacientes ostomizados. Scientia Medica, 18(1), 26-30. 